\title{
COMPLEMENTED SUBSPACES OF PRODUCTS OF BANACH SPACES
}

\author{
PAWEL DOMAŃSKI AND AUGUSTYN ORTYŃSKI
}

\begin{abstract}
It is proved that: (i) every complemented subspace in an infinite product of $L_{1}$-predual Banach spaces $\prod_{i \in I} X_{i}$ is isomorphic to $Z \times \mathbf{K}^{\mathfrak{m}}$, where $\operatorname{dim} \mathbf{K}=1, \mathbf{m} \leq \operatorname{card} I$ and $Z$ is isomorphic to a complemented subspace of $\prod_{i \in J} X_{i}, J \subseteq I, Z$ contains an isomorphic cor. of $c_{0}^{\text {card } J}$; (ii) every injective lcs (in particular, Fréchet) is of the form $Z \times \mathbf{K}^{\mathfrak{m}}, \operatorname{dim} \mathbf{K}=1$, where $Z$ has a fundamental family of seminorms of the cardinality $\mathfrak{r}$ and $Z$ contains an isomorphic copy of $l_{\infty}^{\mathrm{r}}$ (this is a generalization of Lindenstrauss' theorem on injective Banach spaces); (iii) whenever $X \simeq l_{p}, 1 \leq p \leq \infty$, or $X \simeq c_{0}$, then every complemented subspace in a power $X^{\mathfrak{m}}$ ( $\mathfrak{m}$ is an arbitrary cardinal number) is isomorphic to $X^{\mathfrak{r}} \times \mathbf{K}^{\mathfrak{s}}, \mathfrak{r}+s \leq \mathfrak{m}$ (a generalization of the results due to Lindenstrauss and Pelczyński for $\mathfrak{m}=1$ ).
\end{abstract}

\section{INTRODUCTION}

The paper is devoted to the study of complemented subspaces of infinite products of Banach spaces. The first motivation of the paper was a question posed by V. Moscatelli during the ninth Polish-GDR Seminar on Banach Space Theory (Georgenthal, April 1986). He asked about a description of all complemented subspaces of $l_{\infty}^{\mathbf{N}}$. We solve his problem completely, using a method which is similar in spirit to the method applied by Lindenstrauss for characterization of complemented subspaces of $l_{\infty}$ [13, Theorem 2.a.7]. Using similar methods we get a complete description of complemented subspaces of infinite powers of $l_{p}(1 \leq p \leq \infty)$ and $c_{0}$. It turns out that the only such subspaces are the obvious ones. This result generalizes the well-known description of complemented subspaces of $l_{p} \quad(1 \leq p<\infty)$ and $c_{0}$ due to Pelczyński [13, Theorem 2.a.3] and the corresponding result for $l_{\infty}$ due to Lindenstrauss [13, Theorem 2.a.7].

Let us recall [13, Definition 3.b.7] that a locally convex space (lcs) $X$ is primary if for each decomposition of it into two direct summands at least one of them is isomorphic to the whole space. It turns out that all considered above products are primary.

Received by the editors April 25, 1987 and, in revised form, April 12, 1988.

1980 Mathematics Subject Classification (1985 Revision). Primary 46A05, 46A06, 46B99, 46M10; Secondary 46A14, 46A45, 46B25.

Key words and phrases. Complemented subspaces, injective spaces, $L_{1}$-preduals, the DunfordPettis property, products of Banach spaces, locally convex spaces. 
The second main purpose of the paper is a characterization of injective spaces. We say that an lcs $X$ is injective in the category $C$ of lcs whenever $X$ belongs to $C$ and for each lcs $Y \in C$ every isomorphic copy of $X$ contained in $Y$ is complemented. If $C$ is the category of all lcs, then we simply say that $X$ is an injective lcs. We need not distinguish between injectivity in the category of Banach, Fréchet or locally convex spaces because, as we will point out below (Lemma 0) each Banach (Fréchet) space injective in the category of Banach (Fréchet) spaces is also injective in the category of lcs.

We cannot expect a good description of injective Fréchet or locally convex spaces because a satisfactory description of Banach injective spaces is not known. One of the most important results on injective Banach spaces [13, Theorem 2.f.3] asserts that such injective spaces contain copies of $l_{\infty}$. We generalize this fact to the general context: an injective space which does not contain $l_{\infty}^{\mathbf{N}}$ is isomorphic either to $Z_{0}$ or to $Z_{0} \times \mathbf{K}^{\mathbf{m}}$ or to $\mathbf{K}^{\mathrm{m}}$, where $Z_{0}$ is an infinitedimensional injective Banach space and $\operatorname{dim} \mathbf{K}=1$. Thus products of lines are the only separable or reflexive injective spaces.

We also study injective spaces in the category of separable spaces. Zippin [19] proved that the only such Banach space is isomorphic to $c_{0}$. We are unable to generalize this beautiful result. We show only that an injective space in the category of separable lcs either contains $c_{0}^{\mathbf{N}}$ or it is isomorphic to a product of one-dimensional spaces and at most one factor $c_{0}$. ${ }^{1}$

Now, we describe the organization of the paper. $\S 1$ is devoted to general results on complemented subspaces of products of Banach spaces. The main result (Theorem 1.6) shows that semi-Montel spaces of that type are isomorphic to products of lines. Let us recall that an lcs is semi-Montel whenever each its bounded set is relatively compact.

In $\S 2$ we deal with complemented subspaces of products of $L_{1}$-predual Banach spaces (i.e. spaces which duals are isometric to suitable spaces $\left.L_{1}(\Omega, \Sigma, \mu)\right)$. In particular, it concerns products of $C(K)$-spaces. We focus our attention at $l_{\infty}(\Gamma)$ and $c_{0}$. We say that a Banach space $X$ has the DunfordPettis property $[12$, p. 182] if each operator from $X$ into another Banach space sends weakly compact sets into precompact sets. It is known [12, Theorem II.4.30] that all $L_{1}$-predual spaces have this property. It allows us to prove the main result (Theorem 2.8) on endomorphisms on products of $L_{1}$-predual spaces. If we apply it to any projection in a power of an $L_{1}$-predual space $X$, we get that each complemented subspace of $X^{\mathrm{m}}$ is of the form $\mathbf{K}^{\mathrm{r}} \times Z$, where $\mathfrak{r} \leq \mathfrak{m}$ and $Z$ contains a copy of $c_{0}^{\mathfrak{s}}, \mathfrak{s}$ is the smallest cardinality of a family of seminorms generating the topology of $Z$. If $X=l_{\infty}(\Gamma)$, then we can take above $l_{\infty}^{\mathrm{s}}$ instead of $c_{0}^{\mathrm{s}}$. These results imply immediately mentioned above

\footnotetext{
${ }^{\prime}$ After the paper had been sent to the Editors, the authors got the letter from V. Moscatelli who informed that slightly weaker but very similar results had been obtained in his joint paper with $G$. Metafune (Complemented subspaces of products and direct sums of Banach spaces, Ann. Mat. Pura Appl. 153 (1989), to appear). Their methods were different but they also obtained similar results for complemented subspaces of countable direct sums of $l_{p}$ and $c_{0}$.
} 
results on injective spaces and complemented subspaces of $l_{\infty}^{\mathrm{m}}$ and $c_{0}^{\mathfrak{m}}$. In the last section (§3) we present (mainly without proof) results on complemented subspaces of products of $l_{p}(\Gamma)$-spaces.

Notations and conventions. We consider only locally convex spaces (lcs). By $\mathbf{K}$ we denote the scalar field (of real or complex numbers). Moreover, we only deal with linear continuous maps. If $U$ is an absolutely convex 0 -neighborhood in $X$, then $\operatorname{ker} U=\bigcap_{n \in \mathbf{N}}(1 / n) U$. For every linear topology $\tau$ we denote by ker $\tau$ the closure of $\{0\}$ in this topology. If $I_{0} \subseteq I$, then we frequently identify $l_{p}\left(I_{0}\right)\left(\prod_{i \in I_{0}} X_{i}\right.$, resp.) with a subspace of $l_{p}(I)\left(\prod_{i \in I} X_{i}\right.$, resp.) consisting of all elements vanishing outside $I_{0}$. Similarly, we identify $X_{i}$ with a subspace of $\prod_{i \in I} X_{i}$ consisting of elements vanishing outside the singleton $\{i\}$. By gothic letters $\mathfrak{m}, \mathfrak{r}, \mathfrak{s}$ we denote cardinal numbers.

The following simple fact will be often used without any reference (cf. [3, Theorem 3]).

Lemma 0. Every lcs injective in the category of Banach or Fréchet spaces is injective in the category of lcs.

Proof. For example, let $X$ be injective in the category of Fréchet spaces. Thus there is an embedding map $j: X \rightarrow l_{\infty}^{\mathbf{N}}(\Gamma)$ for a suitably chosen set $\Gamma$. If $Y \supseteq X$ is an arbitrary lcs, then the Hahn-Banach theorem allows us to extend $j$ to an operator $j_{1}$ on $Y$. Since $X$ is injective in the category of Fréchet spaces there exists an operator $p: l_{\infty}^{\mathrm{N}}(\Gamma) \rightarrow X$ such that $p \circ j=\mathrm{id}_{X}$. Hence the map $p \circ j_{1}: Y \rightarrow X$ is a projection we are looking for.

\section{Semi-Montel and Banach subspaces of PRoduct SPaCeS}

According to Bellenot and Dubinsky [1], a locally convex space $X$ is said to be a quojection space if $X / \operatorname{ker} U$ is locally bounded for every absolute convex 0 -neighbourhood $U$ in $X$. Next, an lcs $E$ is quasinormable (see [9, p. 106] or [10]) if and only if for every 0-neighborhood $V$ and for each $\alpha>0$ there is a bounded set $B$ in $E$ such that $V \subset B+\alpha U$. It follows from [14] (cf. $[18$, p. 372 and the proof of Lemma 5.9]) that every Fréchet quojection space is quasinormable. Moreover, De Wilde [4, Proposition 2] showed that if $X$ and $F$ are lcs and $Q: X \rightarrow F$ is a continuous open linear surjection with a metrizable quasinormable kernel, then for each bounded set $B_{0}$ in $F$ there exists a bounded set $B$ in $X$ so that $Q(B)=B_{0}$. We say that an lcs $X$ has a property $(A)$ whenever there is a bounded set $B \subset X$ such that for every absolutely convex 0 -neighbourhood $U$ in $X$ the set $B+\operatorname{ker} U$ is a 0 -neighborhood in $X$. It should be pointed out that every lcs with the property $(A)$ is a quojection space. Moreover, each Fréchet quojection space has the property $(A)$. Indeed, if $X$ is a Fréchet quojection space, then for every absolutely convex 0 -neighborhood $U$ the natural quotient map $q: X \rightarrow X / \operatorname{ker} U$ has a locally bounded range and its kernel is a Fréchet quojection space (thus quasinormable). Let $\left(U_{n}\right)_{n \in \mathbf{N}}$ be a 0-neighbourhood base in $X$. Then applying the result of De Wilde to 
a bounded 0-neighbourhood $B_{0}$ in $X / \operatorname{ker} U$ we find a bounded set $B_{n} \subset X$ such that $\operatorname{ker} U_{n}+B_{n}=q^{-1}\left(B_{0}\right)$ is a 0 -neighbourhood in $X$. It is known that we can find a bounded set absorbing all $B_{n}$ 's. Of course, $\operatorname{ker} U_{n}+B$ is a 0 -neighbourhood for every $n \in \mathbf{N}$.

It is obvious that every locally bounded space has the property $(A)$; moreover, we have

Lemma 1.1. An lcs $X$ with a continuous norm has the property $(A)$ iff it is locally bounded.

Proof. Let $U=\{x \in X:\|x\| \leq 1\}$, where \|\| is a continuous norm in $X$. Since $\operatorname{ker} U=\{0\}$, there is a bounded 0 -neighbourhood in $X$.

The class of lcs with the property $(A)$ has several useful permanence properties.

Lemma 1.2. A product of a family of lcs with the property $(A)$ has the property (A) as well.

Proof. Let $X=\prod_{i \in I} X_{i}$, where $X_{i}$ are lcs with the property $(A)$ for every $i \in I$. Let us consider a 0 -neighbourhood $U$ of the form

$$
U=\prod_{k=1}^{n} U_{i_{k}} \times \prod_{i \in I \backslash J} X_{i},
$$

where $J=\left\{i_{1}, i_{2}, \ldots, i_{n}\right\} \subset I, n \in \mathbf{N}$, and $U_{i_{1}}, U_{i_{2}}, \ldots, U_{i_{n}}$ are absolutely convex 0 -neighbourhoods in $X_{i_{1}}, X_{i_{2}}, \ldots, X_{i_{n}}$, respectively. By the definition, there are bounded sets $B_{i}$ in $X_{i}$, such that for every $k=1, \ldots, n$,

$$
B_{i_{k}}+\operatorname{ker} U_{i_{k}}
$$

is a 0 -neighbourhood in $X_{i_{k}}$. It is easily seen that $\operatorname{ker} U+\prod_{i \in I} B_{i}$ is a 0 neighbourhood in $X$ and $\prod_{i \in I} B_{i}$ is a bounded set.

Lemma 1.3. Every quotient (in particular, every complemented subspace) of lcs with the property $(A)$ has the same property.

Proof. Let $X$ be an lcs with the property $(A)$. We denote by $q$ the natural quotient map $q: X \rightarrow X / Y$, where $Y$ is a closed subspace of $X$. There is a bounded set $B$ in $X$ such that for every absolutely convex 0 -neighbourhood $U$ in $X / Y$ the set $B+\operatorname{ker}\left(q^{-1}(U)\right)$ is a 0 -neighbourhood in $X$. Let us observe that

therefore

$$
\operatorname{ker}\left(q^{-1}(U)\right)=q^{-1}(\operatorname{ker} U),
$$

$$
q(B)+q\left(\operatorname{ker}\left(q^{-1}(U)\right)\right)=q(B)+\operatorname{ker} U .
$$

This completes the proof because $q(B)$ is a bounded set.

In general, a subspace of a space with the property $(A)$ need not have this property (for example, a nonlocally bounded subspace with a continuous norm in a product of Banach spaces, cf. Lemmas 1.1 and 1.2). Nevertheless we get the following. 
Lemma 1.4. If an lcs $X$ has the property $(A)$, then for every absolute convex 0-neighbourhood $U$ in $X$ a subspace $\operatorname{ker} U$ has the property $(A)$ as well.

Proof. Since $X$ has the property $(A)$, there is a bounded set $B \subset X$ such that $B+\operatorname{ker} V$ is a 0 -neighborhood in $X$ for every absolutely convex 0 neighbourhood $V$ in $X$ contained in $U$. Hence

$$
(B+\operatorname{ker} V) \cap \operatorname{ker} U=(B \cap \operatorname{ker} U)+\operatorname{ker} V
$$

is a 0 -neighbourhood in $\operatorname{ker} U$.

The next lemma concerns semi-Montel spaces with the property $(A)$.

Lemma 1.5. If a semi-Montel lcs $X$ has the property $(A)$, then its topology coincides with the weak topology.

Proof. If $U$ is an absolutely convex 0 -neighbourhood in $X$, then there exists a compact set $B \subset X$ such that $B+\operatorname{ker} U$ is a 0 -neighbourhood. Therefore $X / \operatorname{ker} U$ is locally compact, i.e. finite dimensional. Finally, every 0 neighbourhood $U$ in $X$ contains a finite codimensional subspace and $U$ contains a weak 0 -neighbourhood.

Now, we are ready to prove the main result of $\S 1$.

Theorem 1.6. Let $X=\prod_{i \in I} X_{i}$ be a product of an arbitrary infinite family of Banach spaces. For a semi-Montel lcs $Y$ the following assertions are equivalent:

(a) $Y$ is isomorphic to $\mathbf{K}^{J}$, where card $J \leq \operatorname{card} I$;

(b) $Y$ is isomorphic to a complemented subspace of $X$;

(c) there is a complemented subspace $Z$ of $X$ and an absolutely convex 0 -neighborhood $U$ in $Z$ such that $\operatorname{ker} U$ is isomorphic to $Y$.

The implications (a) $\Rightarrow$ (b) $\Rightarrow$ (c) are trivial, thus it is enough to prove (c) $\Rightarrow(\mathbf{a})$.

Proof. (c) $\Rightarrow(\mathrm{a})$ : By Lemmas 1.1 and 1.2 every product of Banach spaces has the property $(A)$. Lemma 1.3 implies that $Z$ has the property $(A)$ and, moreover, it follows from Lemma 1.4 that $\operatorname{ker} U$ has the same property. As a consequence of Lemma 1.5 we get that the topology of $Y$ (which is isomorphic to $\operatorname{ker} U$ ) coincides with its weak topology. Obviously, $\operatorname{ker} U$ (and thus $Y$ ) is complete but weakly complete lcs are isomorphic to $\mathbf{K}^{J}$. If card $J \geq \aleph_{0}$, then cardinality of $J$ is equal to the smallest cardinality of a 0 -neighbourhood base of $\mathbf{K}^{J}$. Thus card $J \leq \operatorname{card} I$, because $I$ is an infinite set.

Now, we need the following definition. Let $X$ be an lcs, then the weight of $X, w(x)$, is equal to the least cardinal number $m$ such that there is a family $S$ of seminorms generating the toplogy of $X$ with $\operatorname{card} S=\mathfrak{m}$. It is obvious that the only possible finite value of $w(X)$ is 1 . Similarly, we define the weight of a locally convex topology which need not be Hausdorff.

The first lemma is obvious. 
Lemma 1.7. Let $X, Y$ be locally convex spaces and let $T: X \rightarrow Y$ be an operator. For each locally convex topology $\tau$ on $Y$ nonstronger than the original one, $w(\tau) \leq \mathfrak{m}$, there exists a locally convex topology $\tau_{1}$ on $X$ nonstronger than the original one, $w\left(\tau_{1}\right) \leq \mathfrak{m}$, such that $T\left(\operatorname{ker} \tau_{1}\right) \subseteq \operatorname{ker} \tau$.

Remark. If we consider two products $X=\prod_{i \in I} X_{i}, Y=\prod_{j \in J} Y_{j}$ of Banach spaces, then, for each subset $L \subseteq J, \prod_{j \in J \backslash L} Y_{j}$ is a kernel of a locally convex topology $\tau$ nonstronger than the original one with $w(\tau) \leq \operatorname{card} L$. Thus our Lemma implies that for every operator $T: X \rightarrow Y$ there exists a subset $K \subseteq I$, such that $T\left(\prod_{i \in I \backslash K} X_{i}\right) \subseteq \prod_{j \in J \backslash L}$, and, moreover, if $L$ is finite, then $K$ can be chosen finite but if $L$ is infinite, then $K$ can be chosen with $\operatorname{card} K \leq \operatorname{card} L$.

The next result is known, at least in the most interesting case $\mathfrak{m}=\aleph_{0}$ (i.e., for a Banach space $Y$ ), see [3, Proposition 3].

Proposition 1.8. Let $\mathfrak{m}$ be an infinite cardinal number. Then an lcs $Y, w(Y)<$ $\mathfrak{m}$, is isomorphic to a complemented subspace of an arbitrary product $X=$ $\prod_{i \in I} X_{i}$ of Banach spaces if and only if $Y$ is isomorphic to a complemented subspace of a subproduct $\prod_{i \in J} X_{i}, J \subseteq I$, card $J<\mathfrak{m}$.

Proof. It is enough to prove the "only if" part. If $J \subseteq I$, then we denote by $Q_{J}: X \rightarrow \prod_{i \in J} X_{i}=X_{J}$ the natural projection. It is easily seen that for every subspace $Y$ of $X, w(Y)<\mathfrak{m}$, there is a subset $J_{0} \subseteq I$, card $J_{0}<\mathfrak{m}$, such that for each $J \supseteq J_{0}$, an operator $\left.Q_{J}\right|_{Y}$ is an isomorphism. Let $P: X \rightarrow X$ be a projection onto $Y$. By the remark after Lemma 1.7, there is $J, J_{0} \subseteq J \subseteq I$ with card $J<\mathfrak{m}$, such that

$$
P\left(\prod_{i \in I \backslash J} X_{i}\right) \subseteq \prod_{i \in I \backslash J_{0}} X_{i},
$$

thus

$$
P\left(\operatorname{ker} Q_{J}\right) \subseteq \prod_{i \in I \backslash J_{0}} X_{i} .
$$

Now, let us observe that

$$
P=P Q_{J}+P\left(\mathrm{id}-Q_{J}\right)=P Q_{J}
$$

because

$$
P\left(\mathrm{id}-Q_{J}\right) \subseteq \operatorname{ker} Q_{J_{0}} \cap Y=\{0\} .
$$

Finally, we can define

$$
P_{0}=Q_{J} P
$$

This is a projection because

$$
P_{0}^{2}=Q_{J} P Q_{J} P=Q_{J} P P=P_{0} .
$$

Moreover,

$$
P_{0}(X)=Q_{J}(P(X))=Q_{J}(Y) \subseteq \prod_{i \in J} X_{i},
$$

and this completes the proof. 
The last part of this section will be devoted to injective spaces.

The next lemma is obvious (see, for example, [5, Theorem 1.2(a)]).

Lemma 1.9. If $\left(Y_{i}\right)$ is a family of injective lcs, then $\prod_{i \in I} Y_{i}$ is an injective space as well. $2^{\kappa_{0}}$.

The same holds for injective spaces in the category of separable lcs if card $I \leq$

We mentioned before that every injective space in the category of Banach spaces is an injective lcs. Now, we recall the theorem [3, Theorem 2] that the only infinite-dimensional injective space in the category of separable Banach spaces, i.e. $c_{0}$ (cf. [13, Theorem 2.f.5] and [19]), is an injective space in the category of separable lcs.

We say that an lcs $X$ is transseparable [4, 6] (in [8, p. 67] such spaces are called "seminorm separable") if and only if for every 0 -neighbourhood $U$ in $X$ there is a countable subset $A$ of $X$ such that $A+U=X$.

Let us point out that every separable lcs is transseparable, for metrizable spaces these two notions coincide. Every product of transseparable spaces is trasseparable.

Proposition 1.10. Suppose that a transseparable lcs $X$ contains a subspace $Y$ isomorphic to $c_{0}^{\mathfrak{m}}$ for some cardinal number $\mathfrak{m}$. Then $Y$ is complemented in $X$.

Proof. First, we prove our result for $\mathfrak{m}=1$. Let $\left(U_{i}\right)_{i \in I}$ be a 0 -neighbourhood base of $X$ consisting of absolutely convex sets which intersections with $Y$ are bounded sets. Suppose $X_{i}$ is the completion of the Hausdorff associated space of $X$ equipped with the gauge functional of $U_{i}$ as a seminorm. Of course, by transseparability of $X$, the spaces $X_{i}$ are separable.

There is the standard procedure which allows us to identify $X$ with a subspace of $\prod_{i \in I} X_{i}$. Let $p_{i}: \prod_{i \in I} X_{i} \rightarrow X_{i}$ be the natural projection. It is easily seen that $p_{i}(Y)$ is complemented in $X_{i}$. Thus $Y_{1}=\prod_{i \in I} P_{i}(Y)$ is complemented in $\prod_{i \in I} X_{i}$ and $Y$ can be identified with the diagonal of $Y_{1}$. Since the diagonal is complemented in a product of identical spaces, this completes the proof of $\mathfrak{m}=1$.

The same arguments as in Lemma 1.9 imply that an arbitrary power of $c_{0}$ is complemented in every transseparable space containing it.

Remark. Similarly as in Lemma 0, we can prove that every Fréchet space injective in the category of separable Fréchet spaces is injective in the category of all separable lcs.

\section{COMPLEMENTED SUBSPACES IN PRODUCTS OF $L_{1}$-PREDUAL SPACES}

In this section we need a sequence of lemmas. Since some of them are of their own interest we decided to present them in a slightly more general form than it was necessary. 
Lemma 2.1. Let $\left(I_{k}\right)_{k \in K}$ be a family of infinite sets of the same cardinality and such that card $K \leq \operatorname{card} I_{k}$. Let $X=\prod_{k \in K} \prod_{i \in I_{k}} X_{i}$ be an infinite product of Banach spaces and let $Y$ be an lcs. If $T: X \rightarrow Y$ is a continuous linear operator such that $\left.T\right|_{X_{i}}$ is an isomorphism for every $i \in I_{k}$ and every $k \in K$, then there exists a family of sets $\left(H_{k}\right)_{k \in K}, H_{k} \subseteq I_{k}$, card $H_{k}=$ card $I_{k}$, such that the map $\left.T\right|_{Z}$ is an isomorphism, where $Z=\prod_{k \in K} \prod_{i \in H_{k}} X_{i}$.

Remark. We will use this lemma only for one element set $K$.

Proof. We may assume that $Y=\prod_{l \in L} Y_{1}$, where $Y_{1}$ are Banach spaces.

Let $\alpha$ be the smallest ordinal such that

$$
\operatorname{card} \alpha=\operatorname{card} I_{k}=\operatorname{card}\left(\bigcup\left\{I_{k}: k \in K\right\}\right) .
$$

It is well known (see, for example, [17]) that the set $\{\beta: \beta<\alpha\}$ can be divided into a family of pairwisely disjoint subsets $\left(B_{k}\right)_{k \in K}$ of the cardinality equal to card $\alpha$. Now, we define simultaneously a transfinite sequence $\left(i_{\beta}\right)_{\beta<\alpha}$ of different elements of $I=\bigcup\left\{I_{k}: k \in K\right\}$ and a transfinite increasing sequence $\left(L_{\beta}\right)_{\beta<\alpha}$ of subsets of $L$ such the following conditions are satisfied:

(i) if $X_{\beta}$ is a subspace of $\prod_{\gamma<\beta} X_{i_{\beta}}$ of all finitely nonzero elements, then $\left.T\right|_{X_{\beta}}$ is an isomorphism;

(ii) the natural projection $Q_{\beta}: Y \rightarrow \prod_{l \in L_{\beta}} Y_{1}$ restricted to $Y_{\beta}=\operatorname{lin}\left\{T\left(X_{i_{i}}\right)\right.$ : $\gamma<\beta\}$ is an isomorphism;

(iii) $Q_{\gamma}\left(T\left(X_{i_{\beta}}\right)\right)=\{0\}$ for $\gamma \leq \beta$;

(iv) $\operatorname{card} L_{\beta}<\aleph_{0}$ or $\operatorname{card} L_{\beta} \leq \operatorname{card} \beta$;

(v) if $\beta \in B_{k}$, then $i_{\beta} \in I_{k}$.

As it should be expected, we use the transfinite induction and define $H_{k}=$ $\left\{i_{\beta}: \beta<\alpha, \beta \in B_{K}\right\}$.

Let $L_{0} \subseteq L$ be a finite set and let $Q_{0}$ be the corresponding projection. Applying the remark after Lemma 1.7 we can find $i_{0} \in B_{k}$, where $0 \in B_{k}$, such that $T\left(Y_{i_{0}}\right) \subseteq \operatorname{ker} Q_{0}$.

Now, let us assume that $\left(i_{\beta}\right)$ and $\left(L_{\beta}\right)$ are chosen and the conditions (i) $-(\mathrm{v})$ are fulfilled for $\beta<\beta_{0}$.

First, we show that (i) holds for $\beta=\beta_{0}$. Let

$$
\begin{gathered}
R_{i}: Y_{\beta} \rightarrow T\left(X_{i_{i}}\right), \\
S_{\gamma}: Y_{\beta} \rightarrow \operatorname{lin}\left\{T\left(X_{i_{j}}\right): \delta<\gamma\right\}, \quad \gamma+1 \leq \beta,
\end{gathered}
$$

be linear maps defined as follows:

$$
R_{\gamma}(z)=z_{y}, \quad S_{i,}(z)=\sum_{\delta<i} z_{\delta},
$$

where $z=\sum_{\delta<\beta} z_{\delta}$ has finitely many nonzero terms.

Let us observe that each $z \in Y_{\beta}$ has exactly one representation of the form $z=\sum_{\delta<\beta} z_{\delta}, z_{\delta} \in T\left(X_{i_{\delta}}\right)$. Indeed, otherwise there would be a representation 
of zero with at least two nonzero entries. Thus if $\delta$ were the minimal number of a nonzero entry in the above mentioned representation, then $\delta+1<\beta$ by (iii)

$$
Q_{\delta+1}\left(z_{\delta}\right)=0
$$

and by (ii) $z_{\delta}=0$; a contradiction.

Therefore $\left.T\right|_{X_{\beta}}$ is a continuous algebraic isomorphism onto $Y_{\beta}$ and $R_{\gamma}, S_{\gamma}$ are well defined. Moreover, $R_{\gamma}$ and $S_{\gamma}$ are continuous. Indeed, suppose that a net $\left(z^{a}\right)_{a \in A}$ tends to zero in $Y_{\beta}$. Then $Q_{\gamma} \circ S_{\gamma}\left(z^{a}\right)=Q_{\gamma}\left(z^{a}\right)$ tends to zero too. Since $S_{\gamma}\left(Y_{\beta}\right)=Y_{\gamma}$, (ii) implies that $S_{\gamma}\left(z^{a}\right) \rightarrow 0$. Hence $S_{\gamma}$ and $R_{\gamma}=S_{\gamma+1}-S_{\gamma}$ are continuous.

It is easily seen that the original topology on $X_{\beta}$ is the weakest one which makes all natural projections $P_{i_{\gamma}}: X_{\beta} \rightarrow X_{i_{\gamma}}, \gamma<\beta$, continuous. Since $R_{\gamma} \circ T=$ $T \circ P_{i,}$, for $\gamma<\beta$, so $T$ is an isomorphism on $X_{\beta}$. Thus (i) is fulfilled for $\beta=\beta_{0}$.

As a consequence of (i) we get that $w\left(Y_{\beta}\right)=w\left(X_{\beta}\right) \leq \beta$. Thus there is a set $L_{\beta}$ satisfying (iv) (and containing $L_{\gamma}$ for $\gamma<\beta$ ) such that (ii) is fulfilled. Let $\beta$ belongs to $B_{k}$. The kernel of $Q_{\beta}$ is contained in $\bigcap_{\gamma<\beta} \operatorname{ker} Q_{\gamma}$ and, moreover, there is at least one $X_{i}, i \in I_{k}$, (see the remark after Lemma 1.7) such that $T\left(X_{i}\right) \subset \operatorname{ker} Q_{\beta}$. We choose one of those $i$ as $i_{\beta}$.

The inductive construction is finished. Obviously, the condition (i) holds for $\beta=\alpha$ as well (see the proof above). Since $\overline{X_{\alpha}}=\prod_{k \in K} \prod_{i \in H_{k}} X_{i}$, so $T$ is an isomorphism on $Z$, whenever $H_{k}=\left\{i_{\gamma}: \gamma<\alpha, \gamma \in B_{k}\right\}$ and $Z=\overline{X_{\alpha}}$. This completes the proof.

Remark. It should be pointed out that Lemma 2.1 implies that every complemented subspace $Z$ of a product of Banach spaces contains an isomorphic copy of $\mathbf{K}^{w(Z)}$.

Lemma 2.2. Let $X$ be a Banach space with the Dunford-Pettis property and let $Z$ be an lcs. Then every weakly compact operator $T: X \rightarrow Z$ sends weakly compact sets into precompact sets.

Proof. We may assume that $Z=\prod_{i \in I} Z_{i}$, where $Z_{i}$ are Banach spaces. If $p_{i}: Z \rightarrow Z_{i}$ is the natural projection, then $p_{i} \circ T$ (as a weakly compact map from a Banach space with the Dunford-Pettis property into another Banach space) sends weakly compact sets into precompact sets. Therefore, if $W \subseteq X$ is a weakly compact set, then $p_{i} \circ T(W)$ is a precompact set for $i \in I$. By [7, Theorem 8.3.3],

$$
W_{0}=\prod_{i \in J} p_{i} \circ T(W)
$$

is precompact as well. Since $T(W) \subseteq W_{0}$ so $T(W)$ is precompact.

Lemma 2.3. Let $X=\prod_{i \in I} X_{i}$ be a product of Banach spaces with the DunfordPettis property, let $Z$ be an lcs and let $T: X \rightarrow Z$ be a continuous linear operator. 
If $\left.T\right|_{X_{i}}$ is weakly compact for every $i \in I$, then $T$ sends weakly compact sets into precompact sets.

Proof. We may assume that $Z=\prod_{j \in J} Z_{j}, Z_{j}$ are Banach spaces. Let $p_{j}: Z \rightarrow$ $Z_{j}$ be natural projections. For an arbitrary 0-neighbourhood $U$ in $Z$ let us choose a 0 -neighborhood $V$ in $X$ satisfying $T(V) \subseteq U$. Now, for a weakly compact set $B \subseteq X$ there is a weakly compact set $A \subseteq \prod_{i \in I_{0}} X_{i}, I_{0}$ is a finite subset of $I$, such that $B \subseteq A+U$. Then

$$
T(B) \subseteq T(A)+T(U) \subseteq T(A)+V .
$$

Since Lemma 2.2 implies that $T(A)=\left(\left.T\right|_{\Pi_{i \in I_{0}} X_{i}}\right)(A)$ is precompact, so is $T(B)$.

Lemma 2.4. Let $X=\prod_{i \in I} X_{i}$ be a product of Banach spaces and let $Z$ be a complete lcs. If $T: X \rightarrow Z$ is a continuous linear operator such that $\left.T\right|_{X_{i}}$ is weakly compact for every $i \in I$, then $T$ maps bounded sets into relatively weakly compact sets.

Proof. Because $Z$ can be embedded as a closed subspace of a product of Banach spaces, thus we may assume that $Z$ is equal to such a product $\prod_{j \in J} Z_{j}$. Let $p_{j}: Z \rightarrow Z_{j}$ be a natural projection and let $B_{i} \subseteq X_{i}$ be bounded subsets. There is a 0 -neighborhood $U$ in $X$ such that $p_{j} \circ T(\operatorname{ker} U)=\{0\}$, moreover, there is a finite set $I_{0} \subseteq I$ such that

$$
B=\prod_{i \in I} B_{i} \subseteq \prod_{i \in I_{0}} B_{i}+\operatorname{ker} U .
$$

Hence the set

$$
p_{j} \circ T(B)=p_{j} \circ T\left(\prod_{i \in I_{0}} B_{i}\right)
$$

is relatively weakly compact for every $j \in J$. Finally, $T(B) \subseteq \prod_{j \in J} p_{j} \circ T(B)$ is also relatively weakly compact.

The next lemma is a generalization to the nonlocally bounded case of the known result for Banach spaces (see [12, p. 183 and Theorem II.4.32]).

Lemma 2.5. Let $X$ be an $L_{i}$-predual Banach space and let $Z$ be a complete lcs. If a map $T: X \rightarrow Z$ is not weakly compact, then there is a subspace $Y \subseteq X$ isomorphic to $c_{0}$ such that $\left.T\right|_{Y}$ is an isomorphism.

Proof. Let $j: Z \rightarrow \prod_{i \in I} Z_{i}=Z_{0}$ be an embedding into a product of Banach spaces and let $p_{i}: \prod_{i \in I} Z_{i} \rightarrow Z_{i}$ be the natural projection for every $i \in I$. Suppose $U$ is the unit ball in $X$. If for every $i \in I$ an operator $p_{i} \circ j \circ T$ were weakly compact, then $j \circ T(U)$ would be contained in a weakly compact set

$$
W=\prod_{i \in I} \overline{p_{i} \circ j \circ T(U)} \cap j(Z),
$$

because $j(Z)$ is closed (thus weakly closed) subspace of $Z_{0}$. Therefore if $T: X \rightarrow Z$ is not weakly compact, then there exists $i_{0} \in I$ such that $p_{i_{0}} \circ j \circ T$ 
is not weakly compact either. By the known results on $L_{1}$-predual spaces cited above, there is a subspace $Y$ of $X$ isomorphic to $c_{0}$ for which $\left.p_{i_{0}} \circ j \circ T\right|_{Y}$ is an isomorphism. Obviously, $\left.T\right|_{Y}$ is an isomorphism as well.

Similarly, applying the known fact [13, Proposition 2.f.4] that every nonweakly compact operator from $l_{\infty}(\Gamma)$ into a Banach space $Z$ is an isomorphism on a copy of $l_{\infty}$ contained in the domain space, we are able to prove:

Lemma 2.6. Let $Z$ be a complete lcs. If $T: l_{\infty}(\Gamma) \rightarrow Z$ is not a weakly compact map, then there exists a subspace $Y$ of $l_{\infty}(\Gamma)$ isomorphic to $l_{\infty}$ such that $\left.T\right|_{Y}$ is an isomorphism.

Now, we prove our "key" lemma.

Lemma 2.7. Let $X=\prod_{i \in I} X_{i}$ be a product of Banach spaces with the DunfordPettis property. If $T: X \rightarrow X$ satisfies the following condition for some infinite cardinal number $\mathfrak{m}$ :

$(*)_{\mathfrak{m}}$ there is a subset $J$ of $I$, card $I \backslash J<\mathfrak{m}$, such that $\left.T\right|_{X_{i}}$ is weakly compact for every $i \in J$;

then there exists a subset $H$ of $I$, card $I \backslash H<\mathfrak{m}$, such that $\left.T^{2}\right|_{X_{H}}, X_{H}=$ $\prod_{i \in H} X_{i}$, maps bounded sets into precompact sets.

Proof. According to the remark after 1.7, let us select subsets $H \subseteq J \subseteq I$, card $I \backslash H<\mathfrak{m}$, such that $T\left(X_{H}\right) \subseteq X_{J}$, where $X_{J}=\prod_{i \in J} X_{i}$. We apply Lemma 2.4 to $\left.T\right|_{X_{H}}$ and Lemma 2.3 to $\left.T\right|_{X_{J}}$.

Now, we are ready to prove the main result.

Theorem 2.8. Let $X=\prod_{i \in I} X_{i}$ be an infinite product of $L_{1}$-predual Banach spaces. For every endomorphism $T: X \rightarrow X$ one of the following two conditions is fulfilled:

(a) there is a subset $J \subseteq I$, card $I \backslash J<$ card $I$, such that $T^{2}$ restricted to $\prod_{i \in J} X_{i}$ maps bounded sets into relatively compact sets:

(b) for each subset $J \subseteq I$, card $I \backslash J<\operatorname{card} I$, there is a subspace $Y$ of $\prod_{i \in J} X_{i}$ isomorphic to $c_{0}^{\text {card } I}$ such that $\left.T\right|_{Y}$ is an isomorphism.

If all spaces $X_{i}, i \in I$, are of the form $l_{\infty}(\Gamma)$, then we can assume in (b) that $Y$ is isomorphic to $l_{\infty}^{\text {card } I}$.

Proof. Suppose that card $I=\mathfrak{m}$. Let us assume that (a) is not fulfilled. Then Lemma 2.7 implies that $(*)_{m}$ does not hold either, because $L_{1}$-predual Banach spaces have the Dunford-Pettis property [12, Theorem II.4.30]. There is a subset $J$ of $I$, card $J=\mathfrak{m}$, such that $T \circ j_{i}$ is not weakly compact for $i \in J$, where $j_{i}: X_{i} \rightarrow X$ is the standard embedding. Thus, by Lemma 2.5 , we can find a family $\left(Y_{i}\right)_{i \in J}$ of isomoprhic copies of $c_{0}$ and $Y_{i} \subseteq X_{i}$, such that $\left.T\right|_{Y_{i}}$ is an isomorphism for $i \in J$. Now, it is enough to apply Lemma 2.1 to $K=\{0\}$, $I_{0}=J$ and $\left.T\right|_{\Pi_{i \in J} Y_{i}}$. 
In the case $X_{i}=l_{\infty}(\Gamma)$ the proof is even easier, we should apply Lemma 2.6 instead of Lemma 2.5. We omit details.

Theorem 2.8 has many interesting consequences but first we need another lemma. Its proof is rather simple, and similar to the proof of Lemmas 1.2 and 1.3 .

Lemma 2.9. Let $(Z, \delta)$ be a quotient (in particular, a complemented subspace) of an arbitrary product of Banach spaces. Then for every locally convex topology $\tau$ on $Z$ nonstronger than $\delta$ the following inequality holds:

$$
w(Z / \operatorname{ker} \tau, \hat{\delta}) \leq w(\tau),
$$

where $\hat{\delta}$ is a quotient topology induced by $\delta$.

Proof. The above condition is nearly obvious for $Z$ being any product of Banach spaces. Let us assume that our lemma holds for $X$, we will show it for $Z \simeq X / Y$. Let $q: X \rightarrow Z$ be the natural quotient map and let $\tau$ be an arbitrary locally convex topology on $Z$ nonstronger than the original one. Since $\operatorname{ker} q^{-1}(\tau)=q^{-1}(\operatorname{ker} \tau)$, where $q^{-1}(\tau)$ denotes the topology induced on $X$, the following inequalities hold:

$$
w(Z / \operatorname{ker} \tau) \leq w\left(X / \operatorname{ker} q^{-1}(\tau)\right) \leq w\left(q^{-1}(\tau)\right) \leq w(\tau) .
$$

Corollary 2.10. Let $X=\prod_{i \in I} X_{i}$ be an infinite product of $L_{1}$-predual Banach spaces. Every infinite-dimensional complemented subspace $Z$ of $X$ is isomorphic to $Z_{0} \times \mathbf{K}^{\mathrm{m}}$, where $\mathrm{m} \leq \operatorname{card} I$ and $Z_{0}$ is isomorphic to a complemented subspace of a subproduct $\prod_{i \in J} X_{i}, J \subseteq I$, and contains an isomorphic copy of $c_{0}^{\text {card } J}$

If all spaces $X_{i}$ are of the form $l_{\infty}(\Gamma)$, then $Z_{0}$ contains also $l_{\infty}^{\operatorname{card} J}$. Proof. We will use the transfinite induction over card $I$.

For card $I=1$ it is well-known (cf. [12, Proposition II.4.33]). Let us assume that it is proved for $\operatorname{card} I<\mathfrak{m}$. Now, let card $I=\mathfrak{m}$. If $Z$ does not contain $c_{0}^{\mathfrak{m}}$, then the condition (a) in Theorem 2.8 applied for a projection $T: X \rightarrow Z$ must be satisfied. Obviously, $T$ restricted to $Z_{1}=Z \cap \prod_{i \in J} X_{i}$ is the identity, thus $Z_{1}$ is a semi-Montel space.

If card $I \backslash J=\infty$, then, by Lemma 1.7 and remarks after it, there is a decreasing sequence of subsets: $J=J_{0} \supseteq J_{1} \supseteq J_{2} \supseteq \cdots$, such that

$$
T\left(\prod_{i \in J_{n}} X_{i}\right) \subseteq\left(\prod_{i \in J_{n-1}} X_{i}\right)
$$

where $\operatorname{card} I \backslash J_{n}=\operatorname{card} I \backslash J$ and $n=1,2, \ldots$. Therefore for $H=\bigcap_{n \in \mathrm{N}} J_{n}$

$$
T\left(\prod_{i \in H} X_{i}\right) \subseteq \prod_{i \in H} X_{i} \quad \text { and } \quad \operatorname{card} I \backslash H=\operatorname{card} I \backslash J<\operatorname{card} I .
$$


Finally,

$$
Z_{2}=Z \cap \prod_{i \in H} X_{i}
$$

is a complemented semi-Montel subspace of $\prod_{i \in H} X_{i}$. By Theorem 1.6(b), it is isomorphic to $\mathbf{K}^{\mathfrak{r}}$ for a suitable $\mathfrak{r} \leq \operatorname{card} I$, and, by Lemma 1.9, it is complemented in $Z$.

If $\operatorname{card} I \backslash J<\infty$, then, by Theorem 1.6(c), $Z_{1}$ is isomorphic to $\mathbf{K}^{\mathfrak{r}}$ for a suitable $r \leq \operatorname{card} I$. By Lemma $1.9, Z_{2}:=Z_{1}$ is complemented in $Z$.

In both cases, Lemma 2.9 implies easily

$$
w\left(Z / Z_{2}\right) \leq \operatorname{card} I \backslash J<\operatorname{card} I .
$$

Moreover, $Z \simeq \mathbf{K}^{\mathfrak{r}} \times Z_{3}$, where $Z_{3} \simeq Z / Z_{2}$. By Proposition $1.8, Z_{3}$ is isomorphic to a complemented subspace of some product $\prod_{i \in G} X_{i}$, $\operatorname{card} G<\operatorname{card} I=$ $\mathfrak{m}$. The inductive step is finished and this completes the proof in the " $c_{0}$ case". For $l_{\infty}(\Gamma)$ the proof is quite similar.

The next corollary fully solves Moscatelli's problem [16].

Corollary 2.11. For every cardinal number $\mathfrak{m}$, every complemented subspace of $l_{\infty}^{\mathfrak{m}}$ is isomorphic to $l_{\infty}^{\mathfrak{r}} \times \mathbf{K}^{\mathfrak{s}}$, where $\mathfrak{r}+\mathfrak{s} \leq \mathfrak{m}$. In particular, $l_{\infty}^{\mathfrak{m}}$ is primary. Proof. Applying Corollary 2.10 to $X=l_{\infty}^{\mathrm{m}}$, we get that $Z_{0}$ is a complemented subspace of $l_{\infty}^{\text {card } J}$ and contains a complemented (Lemma 1.9) copy of $l_{\infty}^{\text {card } J}$. By Pelczyński's decomposition method, $Z_{0} \simeq l_{\infty}^{\text {card } J}$.

Remark. Obviously, if $\mathfrak{s} \leq \mathfrak{r}$, then $l_{\infty}^{\mathfrak{r}} \times \mathbf{K}^{\mathfrak{s}} \simeq l_{\infty}^{\mathfrak{r}}$.

Every lcs may be embedded into a product of $l_{\infty}(\Gamma)$ spaces for suitable $\Gamma$. It is known that every injective infinite-dimensional Banach space contains a copy of $l_{\infty}$ [13, Theorem 2.f.3]. Hence using similar arguments as in the proof of Corollaries 2.11 and 2.10 we have

Corollary 2.12. Every injective lcs is isomorphic to $\mathbf{K}^{\mathfrak{m}}$ or to $Z \times \mathbf{K}^{\mathfrak{m}}$, where $Z$ contains a copy of $l_{\infty}^{w(Z)}$ and $\mathfrak{m}$ is a suitably chosen cardinal number. In particular, it is isomorphic to $\mathbf{K}^{\mathfrak{m}}$ or it contains a copy of $l_{\infty}$.

Corollary 2.13. Every separable infinite-dimensional injective lcs (Fréchet space) is isomorphic to $\mathbf{K}^{\mathfrak{m}}, \mathfrak{m} \leq \operatorname{card} \mathbf{K}$ (to $\mathbf{K}^{\mathbf{N}}$ ).

Proof. Every separable lcs (Fréchet space) may be embedded into $l_{\infty}^{\text {card } \mathbf{K}}\left(l_{\infty}^{\mathbf{N}}\right)$ because in every separable lcs there is a 0 -neighborhood base cardinality of which does not exceed card $\mathbf{K}$ [7, Theorem 1.5.6]. On the other hand, the product $\mathbf{K}^{\mathfrak{m}}$ for $\mathfrak{m} \leq$ card $\mathbf{K}$ is separable [7, Corollary 2.3.16]. Hence Corollary 2.12 implies our result.

We can prove an analogue of Corollary 2.11 for $c_{0}$.

Corollary 2.14. For every cardinal number $m$ each infinite-dimensional complemented subspace of $c_{0}^{\mathfrak{m}}$ is isomoprhic to $c_{0}^{\mathfrak{r}} \times \mathbf{K}^{\mathfrak{s}}$, where $\mathfrak{r}+\mathfrak{s} \leq \mathfrak{m}$. In particular, $c_{0}^{\mathrm{m}}$ is primary. 
The proof is similar as the proof of Corollary 2.11. We should apply Proposition 1.10 .

Corollary 2.15. Every injective space in the category of separable lcs is either isomorphic to $c_{0} \times \mathbf{K}^{\mathfrak{m}}$ or to $\mathbf{K}^{\mathfrak{m}}$ or to $Z \times \mathbf{K}^{\mathfrak{m}}$, where $\mathfrak{m} \leq$ card $\mathbf{K}, Z$ contains a complemented copy of $c_{0}^{w(Z)}$, and $w(Z)=\infty$.

Proof. By Theorem 1.5.6 and Corollary 2.3.16 in [7], each separable lcs may be embedded into a separable lcs $C(0.1)^{\text {card } \mathbf{K}}$. Every $C(K)$-space is $L_{1}$-predual [12, p. 153], thus we are able to apply Corollary 2.10. This completes the proof because $c_{0}$ is the only one infinite-dimensional Banach space injective in the category of separable lcs (see [19]).

The last corollary is a strong generalization of Palamodov's result [15, Theorem 9.2] on Fréchet-Schwartz injective spaces.

Corollary 2.16. A (separable) semireflexvie lcs is injective (in the category of separable lcs) if and only if it is a product of (at most card $\mathbf{K}$ ) one-dimensional spaces.

Proof. A subspace of a semireflexive lcs is necessarily semireflexive, thus our result is a consequence of Corollaries 2.12 and 2.15.

The above corollaries suggest the following question due to L. Nachbin.

Problem 2.1. Is every injective lcs (Fréchet space) isomorphic to a product of injective Banach spaces? Equivalently, is every complemented subspace of a product of $l_{\infty}(\Gamma)$-spaces isomorphic to a product of Banach spaces?

The authors do not know if there exists a complemented subspace of a product of Banach spaces which is not isomorphic to a product of Banach spaces. ${ }^{2}$

The second problem concerns a "Fréchet analogue" of Zippin's result [19].

Problem 2.2. Is every infinite-dimensional Frèchet space injective in the category of separable lcs isomorphic to one of the following spaces: $c_{0}^{\mathbf{N}}, \mathbf{K}^{\mathbf{N}}$, $c_{0} \times \mathbf{K}^{\mathbf{N}}, c_{0}$ ?

\section{COMPLEMENTED SUBSPACES OF PRODUCTS OF $l_{p}(\Gamma)$-SPACES FOR $1 \leq p<\infty$}

We will assume throughout the section that $1 \leq p<\infty$. The first lemma allows us to strengthen Lemma 2.1.

Lemma 3.1. Let $\left(I_{k}\right)_{k \in K}$ be a family of infinite sets of the same cardinality and such that $\operatorname{card} K \leq \operatorname{card} I_{K}$. Let $X=\prod_{k \in K} \prod_{i \in I_{k}} X_{i}$ be a subspace of locally convex space $Y$. If all $X_{i}, i \in I_{k}, k \in K$, are complemented in $Y$, then there

\footnotetext{
2 After this paper had been prepared the first author proved (Complemented subspaces of products of Hilbert spaces, to appear) that every complemented subspace of an arbitrary product of Hilbert spaces is isomorphic to a product of Hilbert spaces.
} 
exists a family $\left(H_{k}\right)_{k \in K}, H_{k} \subset I_{k}$, card $H_{k}=\operatorname{card} I_{k}$ for $k \in K$, such that $Z=\prod_{k \in K} \prod_{i \in I_{k}} X_{i}$ is also a complemented subspace of $Y$.

Remark. We will use this lemma for a one-element set $K$.

Proof. We will construct $\left(H_{k}\right)_{k \in K}$ by the transfinite induction. Let $\alpha$ be the smallest ordinal such that $\operatorname{card} \alpha=\operatorname{card} I_{k}=\operatorname{card} \bigcup_{k \in K} I_{k}$. Similarly as in the proof of 2.1 , we can divide the set of ordinals $\{\beta: \beta<\alpha\}$ into a family of pairwisely disjoint subsets $\left(B_{k}\right)_{k \in K}, \operatorname{card} B_{k}=\operatorname{card} \alpha$.

If $0 \in B_{k}$, then let us choose an arbitrary $i_{0} \in I_{k}$ and a projection $P_{0}: Y \rightarrow$ $X_{i_{0}}$. Now, we will select a transfinite sequence $\left(i_{\beta}\right)_{\beta<\alpha} \subset \bigcup_{k \in K} I_{k}$ and a transfinite sequence of projections $\left(P_{\beta}\right)_{\beta<\alpha}, P_{\beta}: Y \rightarrow X_{i_{\beta}}$ satisfying the following conditions for every $\beta<\alpha$ :

(i) $P_{\beta} \circ P_{\gamma}=0$ for $\gamma<\beta$;

(ii) $P_{\gamma}\left(X_{i_{\beta}}\right)=0$ for $\gamma<\beta$;

(iii) $i_{\beta} \in I_{k}$ whenever $\beta \in B_{k}$.

Let us assume that $i_{\gamma}$ and $P_{\gamma}$ are fixed for $\gamma<\beta_{0}$ and the conditions (i)(iii) are satisfied for $\beta<\beta_{0}$. Now, we define $P_{\beta_{0}}$ and $i_{\beta_{0}}$. The subspace $\bigcap_{\gamma<\beta_{0}} \operatorname{ker} P_{\gamma}$ is equal to the kernel of a locally convex topology $\tau, w(\tau) \leq \beta_{0}<\alpha$ on $Y$ nonstronger than the original one. Obviously, if $\beta_{0} \in B_{k}$ then it contains some $X_{i_{\beta_{0}}}$ such that $i_{\beta_{0}} \in I_{k}$. Thus $i_{\beta_{0}}$ is fixed.

Let $S_{\beta_{0}}: Y \rightarrow X_{i_{\beta_{0}}}$ be a projection. Then we define

$$
P_{\beta_{0}}=S_{\beta_{0}} \circ\left(\mathrm{id}-\prod_{\gamma<\beta_{0}} P_{\gamma}\right) .
$$

It follows from (i) and (ii) that the product $\prod_{\gamma<\beta_{0}} P_{\gamma}$ is well defined. Thus our construction is finished.

Applying the same arguments as above, a map $P: Y \rightarrow \prod_{\gamma^{\prime<\alpha}} X_{i_{\gamma}}$

$$
P(x)=\prod_{\gamma<c} P_{y}(x),
$$

is the well-defined projection onto. This completes the proof.

The next lemma corresponds to Lemma 2.5.

Lemma 3.2. Let $Z=\prod_{i \in I} l_{p}\left(\Gamma_{i}\right)$. If $T: l_{p}(\Gamma) \rightarrow Z$ is not a compact map, then there is a subspace $Y \subset l_{p}(\Gamma)$ isomorphic to $l_{p}$ such that $T$ restricted to $Y$ is an isomorphism and $T(Y)$ is complemented in $Z$.

Proof. It is easy to see that if $T: l_{p}(\Gamma) \rightarrow Z$ is not compact, then there is a separable subspace $Y_{0}$ of the domain such that $\left.T\right|_{Y_{0}}$ is not compact. Thus without loss of generality, we can assume that $T: l_{p} \rightarrow Z$.

Let $p_{i}: Z \rightarrow l_{p}\left(\Gamma_{i}\right)$ be the natural projection. If all maps $p_{i} \circ T$ were compact, then $T$ would be compact as well. Hence, for some $i \in I, p_{i} \circ T: l_{p} \rightarrow l_{p}\left(\Gamma_{i}\right)$ 
is not compact and, obviously, there is a countable subset $\Gamma_{0} \subset \Gamma_{i}$ such that $p_{i} \circ T\left(l_{p}\right) \subset l_{p}\left(\Gamma_{0}\right)$.

It is well known [13, Proposition 2.a.2] that every infinite-dimensional subspace of $l_{p}$ contains a complemented isomorphic copy of $l_{p}$. Moreover, a noncompact endomorphism on $l_{p}$ is not strictly singular [13, p. 76]. Therefore there exists an isomorphic copy $Y$ of $l_{p}$ contained in $l_{p}$ such that $p_{i} \circ T$ maps $Y$ isomorphically onto a complemented subspace of $l_{p}\left(\Gamma_{0}\right)$.

Obviously, since the operator $\left.p_{i}\right|_{T(Y)}$ is an isomorphism, a map $S: Z \rightarrow T(Y)$ defined as follows:

$$
S(z)=p_{i}^{-1} \circ\left(R \circ p_{i}(z)\right)
$$

is a projection, whenever $R: l_{p}\left(\Gamma_{i}\right) \rightarrow p_{i} \circ T(Y)$ is a projection.

Since the proof of the following two results are quite similar to the proof of Lemma 2.4 and Theorem 2.8 respectively, we omit them.

Lemma 3.3. Let $X=\prod_{i \in I} X_{i}$ be an infinite product of Banach spaces and let $Z$ be an lcs. If $T: X \rightarrow Z$ is a continuous linear operator such that $\left.T\right|_{X_{i}}$ is compact for every $i \in I$, then $T$ maps bounded sets into precompact sets.

Theorem 3.4. Let $X=\prod_{i \in I} l_{p}\left(\Gamma_{i}\right)$ be an infinite product. For every endomorphism $T: X \rightarrow X$ one of the following two conditions is fulfilled:

(a) there is a subset $J \subset i, \operatorname{card}(I \backslash J)<\operatorname{card} I$, such that $T$ restricted to $\prod_{i \in J} l_{p}\left(\Gamma_{i}\right)$ maps bounded sets into relatively compact sets;

(b) for each subset $J \subset I$, $\operatorname{card}(I \backslash J)<\operatorname{card} I$, there exists a subspace $Y$ of $\prod_{i \in J} l_{p}\left(\Gamma_{i}\right)$ isomorphic to $l_{p}^{\mathrm{card} I}$ such that $\left.T\right|_{Y}$ is an isomorphism and $T(Y)$ is complemented in $X$.

Remark. We apply Lemma 3.1 to select $Y$ in (b) such that $T(Y)$ is complemented in $X$.

The following two corollaries are analogues of Corollary 2.10 and Corollaries 2.11 and 2.14 , respectively.

Corollary 3.5. Let $X=\prod_{i \in I} l_{p}\left(\Gamma_{i}\right)$ be an infinite product. Each infinitedimensional complemented subspace $Z$ of $X$ is isomorphic to $Z_{0} \times \mathbf{K}^{\mathrm{m}}$, where $Z_{0}$ is isomorphic to a complemented subspace of some subproduct $\prod_{i \in J} l_{p}\left(\Gamma_{i}\right)$, $J \subset I$, and $Z_{0}$ contains a complemented copy of $l_{p}^{\text {card } . I}$.

Corollary 3.6. For every cardinal number $\mathrm{m}$ each complemented subspace of $l_{p}^{\mathrm{m}}$ is isomorphic to $l_{p}^{\mathfrak{r}} \times \mathbf{K}^{\mathfrak{s}}$, where $\mathfrak{r}+\mathfrak{s} \leq \mathfrak{m}$. In particular, the space $l_{p}^{\mathfrak{m}}$ is primary.

Acknowledgement. The authors are very indebted to Professor L. Drewnowski and Dr. M. Nawrocki for their critical reading of the manuscript and stimulating discussions. 


\section{REFERENCES}

1. S. Bellenot and E. Dubinsky, Fréchet spaces with nuclear Köthe quotients, Trans. Amer. Math. Soc. 273 (1982), 579-594.

2. M. DeWilde, Sur le relevement des parties bornées d'un quotient d'espaces vectoriels topologiques, Bull. Soc. Roy. Sci. Liège 43 (1974), 299-301.

3. J. Diestel and R. H. Lohman, Applications of mapping theorems to Schwartz spaces and projections, Michigan Math. J. 20 (1973), 39-44.

4. P. Domański, On the separable topological vector spaces, Funct. Approx. 14 (1984), 117-122.

5. __. Twisted sums of Banach and nuclear spaces, Proc. Amer. Math. Soc. 97 (1986), 237-243.

6. L. Drewnowski, Another note on Kalton's theorem, Studia Math. 52 (1975), 233-237.

7. R. Engelking, General topology, Monogr. Mat. 60, PWN, Warsaw, 1977.

8. M. G. Garnir, M. DeWilde and J. Schmets, Analyse fonctionnelle, Birkhäuser-Verlag, Basel and Stuttgart, 1968.

9. A. Grothendieck, Sur les espaces $(F)$ et $(D F)$, Summa Brazil. Math. 3 (1954), 57-122.

10. H. J. Junek, Locally convex spaces and operator ideals, Texts in Math. 68, Teubner, Leipzig, 1983.

11. G. Köthe, Topological vector spaces, vol. I, Springer-Verlag, Berlin, Heildelberg and New York, 1969.

12 J. Lindenstrauss and L. Tzafriri, Classical Banach spaces, Lecture Notes in Math., vol. 338, Springer-Verlag, 1973.

13. __ Classical Banach spaces, vol. I, Springer-Verlag, Berlin Heildenberg, and New York, 1977.

14. R. Meise and D. Vogt, A characterization of the quasi-normable Fréchet spaces, Mat. Nachr. 122 (1985), 141-150.

15. V. P. Palamodov, Homological methods in the theory of locally convex spaces, Uspehi Mat. Nauk 26 (1971), 3-66; English transl., Russian Math. Surveys 26 (1971), 1-64.

16. Open problem presented at the Ninth Seminar Poland-GDR on Operator Ideals and Geometry of Banach Spaces, Georgenthal, April 1986, communicated by A. Pietsch, Forschungsergebnisse Friedrich-Schiller Universität Jena, Jena, 1988.

17. W. Sierpiński, Cardinal and ordinal numbers, Monogr. Mat. 34, PWN, Warsaw, 1958.

18. D. Vogt, Some results on continuous linear maps between Fréchet spaces, Functional Analysis: Surveys and Recent Results. III (K.-D. Bierstedt and B. Fuchssteiner, eds.), North-Holland Studies in Math. 90, North-Holland, 1984, pp. 349-381.

19. M. Zippin, The separable extension problem, Israel J. Math. 26 (1977), 372-387.

Institute of Mathematics, A. Mickiewicz University, ul. Matejki 48/49, 60-769 Poznań, Poland 